\title{
Article \\ Cooperativity between Dimerization and Binding Equilibria in the Ternary System Laponite-Indocyanine Green-Water
}

\author{
Giuseppe Cinelli ${ }^{1}$, Gennaro Bufalo ${ }^{2}$, Francesco Lopez ${ }^{1}$ and Luigi Ambrosone ${ }^{3, *}$ \\ 1 Dipartimento di Agricoltura Ambiente Alimenti (DiAAA), University of Molise, 86100 Campobasso, Italy; \\ giuseppe.cinelli@gmail.com (G.C.); lopez@unimol.it (F.L.) \\ 2 INAIL-Sector Research, Certification and Verification, Department of Naples, I-80121 Naples, Italy; \\ g.bufalo@inail.it \\ 3 Department of Medicine and Health Science V. Tiberio, University of Molise, 86100 Campobasso, Italy \\ * Correspondence: ambroson@unimol.it
}

check for

updates

Citation: Cinelli, G.; Bufalo, G.;

Lopez, F.; Ambrosone, L.

Cooperativity between Dimerization

and Binding Equilibria in the Ternary

System Laponite-Indocyanine

Green-Water. ChemEngineering 2021,

5,6. https://doi.org/10.3390/

chemengineering5010006

Received: 22 October 2020

Accepted: 31 December 2020

Published: 1 February 2021

Publisher's Note: MDPI stays neutral with regard to jurisdictional clai$\mathrm{ms}$ in published maps and institutional affiliations.

Copyright: () 2021 by the authors. Licensee MDPI, Basel, Switzerland. This article is an open access article distributed under the terms and conditions of the Creative Commons Attribution (CC BY) license (https:// creativecommons.org/licenses/by/ $4.0 /)$.

\begin{abstract}
Laponite is an artificial nanoclay available in large quantities and at low cost. This marterial represents an efficient and suitable way of delivering hydrophobic vital dyes without the need for chemical functionalization. Laponite is available in large quantities and at low cost, then it would be an efficient way of delivering hydrophobic vital dyes without the need for chemical functionalization. The hydrodynamic diameter of laponite extrapolated to infinite dilution indicates that this clay is completely exfoliated. Furthermore, the hydrodynamic diameter in the laponite-Indocyanine green-water ternary system, at a fixed laponite concentration $(2 \%(\mathrm{~m} / \mathrm{m}))$ exhibits a saturation curve. It was found that the extrapolated diameter at dye zero concentration is smaller than in pure water. Absorption spectra with fixed concentration of dye exhibit a red shift of 10-13 nm. On the contrary, the spectra acquired at a constant concentration of laponite do not undergo any displacement. The deconvolution of the spectra with two Gaussian peaks allows to calculate the concentration of the monomeric and dimeric species. The results were interpreted as a synergy between the dye dimerization balance and the dye-laponite binding.
\end{abstract}

Keywords: laponite; indocyanine green; binding equilibrium; dimerization equilibrium; dynamic light scattering

\section{Introduction}

Laponite (LAP) is an artificial clay, consisting of a three-dimensional network containing two tetrahedral silica interspersed with one octahedral magnesia. This structure gives the LAP molecule the ability to form gel-like structures in aqueous media [1]. In recent years, there has been great demand to use LAP, also khown as artificial hectorite, in biomedical field [2]. The choice of LAP is crucial for in-vitro and in-vivo bio-imaging analyses. In this regard, the research has focused on the study of LAP-dye interactions in order to solubilize a hydrophobic dye without the need for chemical functionalizations [3]. Very recently, chemometric methods are being considered as potential systems in preparing dye biosorption from ternary system for diagnostic and environmental sectors [4-7]. Due to their ability of absorbing light in the visible and near-infrared regions, cyanine dyes are used to color images of biological systems. Among the vital dyes [8], indocyanine green (ICG) is widely used to stain epiretinal membranes during microsurgery. Indeed, this dye exhibits a marked absorption around $800 \mathrm{~nm}$ where blood and other biological tissues are transparent. Such molecule has to be administered as salt solution, however ICG aqueous solutions give rise to side reactions of aggregation and degradation which reduce the performance of the dye [9]. This implies that it must be used quickly, otherwise it loses its effectiveness. Pre-clinical studies performed on animals have shown that ICG molecules are potentially toxic to pre-retinal membranes [10]. 
Although, several studies accounts for many of the physical properties of ICG solutions, a number of phenomena remains unexplained. For example, the correlation between associative ability of dye and its toxicity observed in-vivo experiments. Obviously, if one reduces the dye dosage the success of surgery may be compromised. Alternatively, a nanocarrier may be used for the transportation of the dye to the target site. Recently, we proposed to administer the vital dyes in the form of liposomal dispersions [11]. It was suggested that smectic clays in general and LAP in particular have the characteristics of suitable drug-carriers. It was suggested that smectic clays in general have the characteristics of suitable drug-carriers [12-14]. Particularly, LAP was found to be a good nano-carrier for effectively delivering cancer drugs such as doxorubicin [15]. The particular LAP form consisting of an octahedral magnesia sheet sandwiched between two tetrahedral silica sheets may be exploited for delivering of ICG to a specific tissue. In order to understand the dye delivery mechanism, some properties such as the dielectric constant [16] of the medium, the partial molar volumes [17] or more generally the LAP-ICG-water interactions have to be known. Herein, the LAP-ICG-water interactions are investigated both by dynamic ligth scattering and UV.Vis spectroscopy. The deconvolution of the absorption spectra is used to determine the concentration of the monomeric and dimeric species. To rationalize the results, a model, where the dimerization and binding equilibria cooperate, is suggested.

\section{Materials and Methods}

\subsection{Chemicals}

Laponite (RD) powder identified by the chemical formula $\mathrm{Na}_{0.7}^{+}\left[\mathrm{Si}_{8} \mathrm{Mg}_{5.5} \mathrm{O}_{20}(\mathrm{OH})_{4}\right]^{-0.7}$ was kindly offered by Rockwood Additives BYK. Indocyanine green (ICG) was purchased from Patheon Italia S.p.a., Italy. The commercial product, ICG-PULSION was medical grade compound, i.e., injectable commercial solution and was used without purification.

\subsection{Uv-Vis Spectroscopy}

Spectra, in the 200-900 $\mathrm{nm}$ wavelength range, were recorded at room temperature on a Cary 100 Varian UV-Vis equipped with a thermostated cell of $1 \mathrm{~cm}$ ligth-path.

\subsection{Samples Preparations}

Freshly ultrapure water was used to prepare all the solutions. LAP suspensions were prepared by dispensing LAP powder in milli-pore water and subsequent by stirring for $24 \mathrm{~h}$. ICG solutions were prepared by dilution from a stock solution. The final LAP-IGCWater ternary system were prepared by adding IGC solutions to LAP dispersions at a fixed concentration of $0.25,1 \%, 2 \%$ and $3 \%$. IGC (CICG) concentrations were used in the rage of 2.45-24.5 $\mu \mathrm{M}$.

\subsection{Dynamic Light Scattering}

The average hydrodynamic diameter values of the ternary systems were determined at $25{ }^{\circ} \mathrm{C}$ by means of dynamic light scattering (DLS) and electrophoretic mobility measurements using a Malvern UK Zetasizer-Nano ZS90 commercial instrument operating with a $4 \mathrm{~mW}$ He-Ne laser (633 $\mathrm{nm}$ wavelength). DLS records were collected leaving the instrument free to optimize the instrumental parameters. The size distribution by intensity of scattered light was recovered, using the software implemented by the manufacturer, by taking the inverse Laplace transform of the ACF and subsequent application of Stokes-Einstein equation, assuming the viscosity of the water solution at $25^{\circ} \mathrm{C}$.

\section{Results and Discussion}

\subsection{Z-Average Measurements}

Plate-like particles of sodium laponite consist of an octahedral magnesia sheet sandwiched between two tetrahedral silica sheets. In the tetrahedral sheet, few magnesium atoms are substituted with lithium atoms. This replacement is usually referred to as isomorphic substitution, i.e., an atom of higher valency is replaced by one of lower valency. 
This results in a deficit of positive charge or an excess of negative charge. Thus, the faces of the clay platelets become negatively charged and these negative charges are compensated by counterions $\mathrm{Na}^{+}$. As schematically illustrated in Figure 1 the replacement of $\mathrm{Mg}^{2+}$ with $\mathrm{Li}^{+}$results in an excess of negative charge in the faces of LAP-platelet.

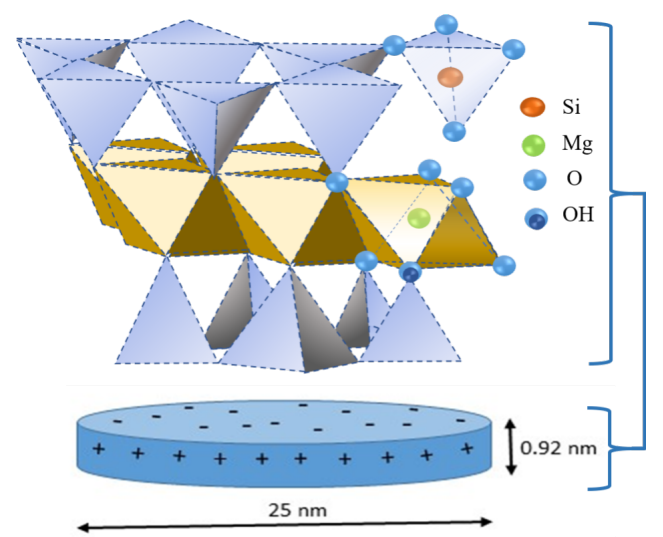

Figure 1. Illustration of the LAP nanoscystal geometry (disk shape) and chemical structure. The circular surfaces of the crystal posses a negative charge due to substitution of $\mathrm{Mg}^{2+}$ by $\mathrm{Li}^{+}$in octahedral layer of the crystal. The edges have a positive charge depending on the protonation of the exposed hydroxyl groups.

Although the shape of LAP particles is not spherical, the quantity Z-average, measured by DLS provide the size of the dynamic solvated particles. In other words, Z-average, is the diameter of a sphere that has the same translational diffusion coefficient as the particle being measured. Such a parameter is experimentally calculated as harmonic intensity weighted arithmetic average particle diameter, i.e.,

$$
d_{z}=\frac{\sum_{i} I_{i}}{\sum_{i} \frac{I_{i}}{d_{i}}}
$$

where $I_{i}$ and $i$ and $d_{i}$ are the scattered intensity and diameter of particle $i$.

In Figure $2, d_{z}$ vs. LAP mass fraction, $W_{L A P}$, is plotted for the binary system LAPwater. It is apparent that the diameter is directly proportional to LAP concentration for mass fraction below 0.03 . This behavior with concentration can be considered as a compromise between those forces which pull molecules together and those which push them apart. To determine the structural propensity of the LAP, it is necessary to have the system where only LAP-solvent interactions exist. This situation is possible at infinite dilution where the LAP-LAP interactions are negligible and predominate LAP-solvent interactions. Since the particles of such a state are separated by an infinite distance, the simplification introduced by assumption of infinite dilution is that the disturbance of the flow of the solvent by given particle does not overlap with the disturbance of flow caused by the presence of a second suspended particle.

Experimental data of Figure 2 show a linear relationship between $d_{z}$ and $W_{L A P}$, then the extrapolation is easily done by fitting the data to a polynomial of degree 1 . The fitting provides $d_{z}^{\infty}=34 \pm 7 \mathrm{~nm}$. This value, practically identical to the diameter of a single LAP disc, indicates that the synthetic clay in the state of infinite dilution is completely exfoliated.

If a small amount of ICG is added the resulting ternary system has a rather different and peculiar hydrodynamic behavior. 


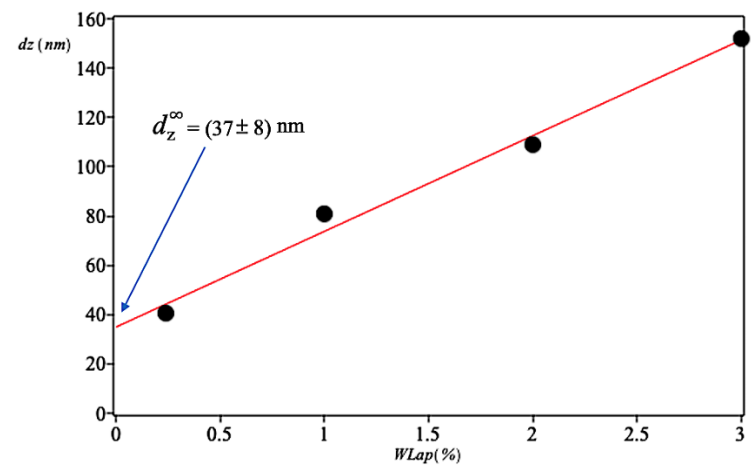

Figure 2. LAP Hydrodynamic diameter in binary mixtures at $25^{\circ} \mathrm{C}$. The extrapolated value at infinite dilution is very close to the diameter of a single LAP disk.

Figure 3 shows $d_{z}$ vs. ICG concentration, $\left(C_{I C G}\right)$, for the ternary system with LAP mass fraction fixed at 0.02 . As one can see, for $C_{I C G}>12 \mu \mathrm{M}, d z=176 \mathrm{~nm}$ remains practically constant, furthermore such value is greater than the corresponding, $d z=109 \mathrm{~nm}$, measured in the binary system LAP-water. For $C_{I C G}<13 \mu \mathrm{M}, d_{z}$ decreases. Interestingly the diameter extrapolated to zero- $C_{I C G} d_{z}^{\infty}$ is significantly lower than the $d_{z}$ measured in the binary system containing $2 \%(\mathrm{w} / \mathrm{w})$ of LAP.

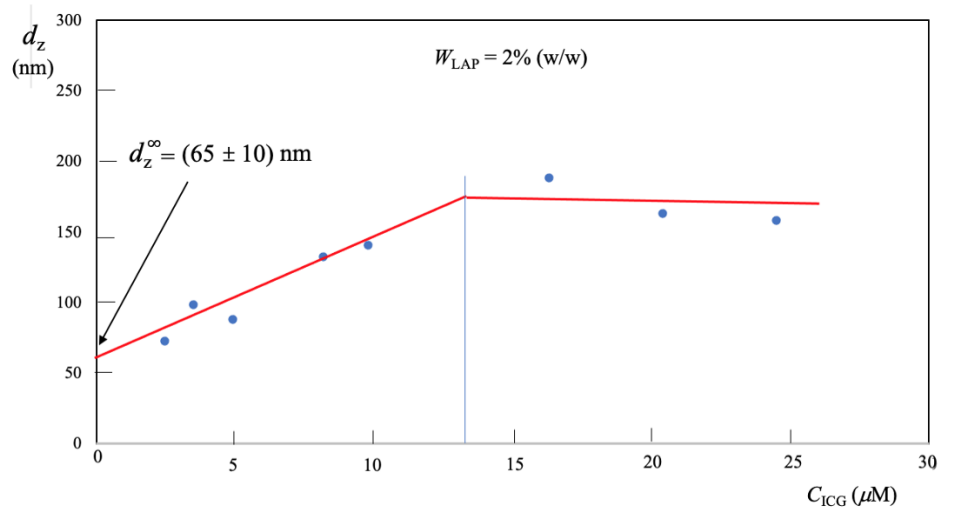

Figure 3. Hydrodynamic LAP diameter in ternary LAP-IGG-water system with fixed mass fraction of LAP $W_{L A P}=0.02$. (the curve is a guide for the eyes).

In order to correctly interpret results of Figure 3, we recall that the experiments were performed with commercial ICG, surgically used for tissue perfusion. This is a saline solution for infusion, therefore an increase in the concentration of dye induces an increase in ionic strength. A higher ionic strength causes an aggregation of ICG via $\pi-\pi$ stacking, hence an increase in $d_{z}$ is observed [18].

\subsection{Absorption Spectra}

A ICG molecule contains conjugated system with alternating double bonds. Accordingly, electrons in the $\pi$-bonds are delocalized on the entire conjugated chain. Depending on the length of conjugated chain, absorption bands appear in the different energy of the UV-Visible spectral region.

Since ICG molecules are predict to be planar molecule owing to the extensive conjugation throughout the molecules, it is possible to stack them on top of each other $[19,20]$. Upon the formation of dimer, molecular orbitals interact and a new peak is created. Thus, in pure water ICG absorption spectrum essentially presents two peaks. The first centered at $780 \mathrm{~nm}$ attributable to the monomer (M-band), the second centered at $715 \mathrm{~nm}$ constitutes the dimer band (D-Band) [21]. In Figure 4, ICG absorption spectra, recorded both in binary systems ICG-water and in ternary system LAP-ICG-water with 1, 2 or 3\%, are plotted for each ICG concentration investigated. 

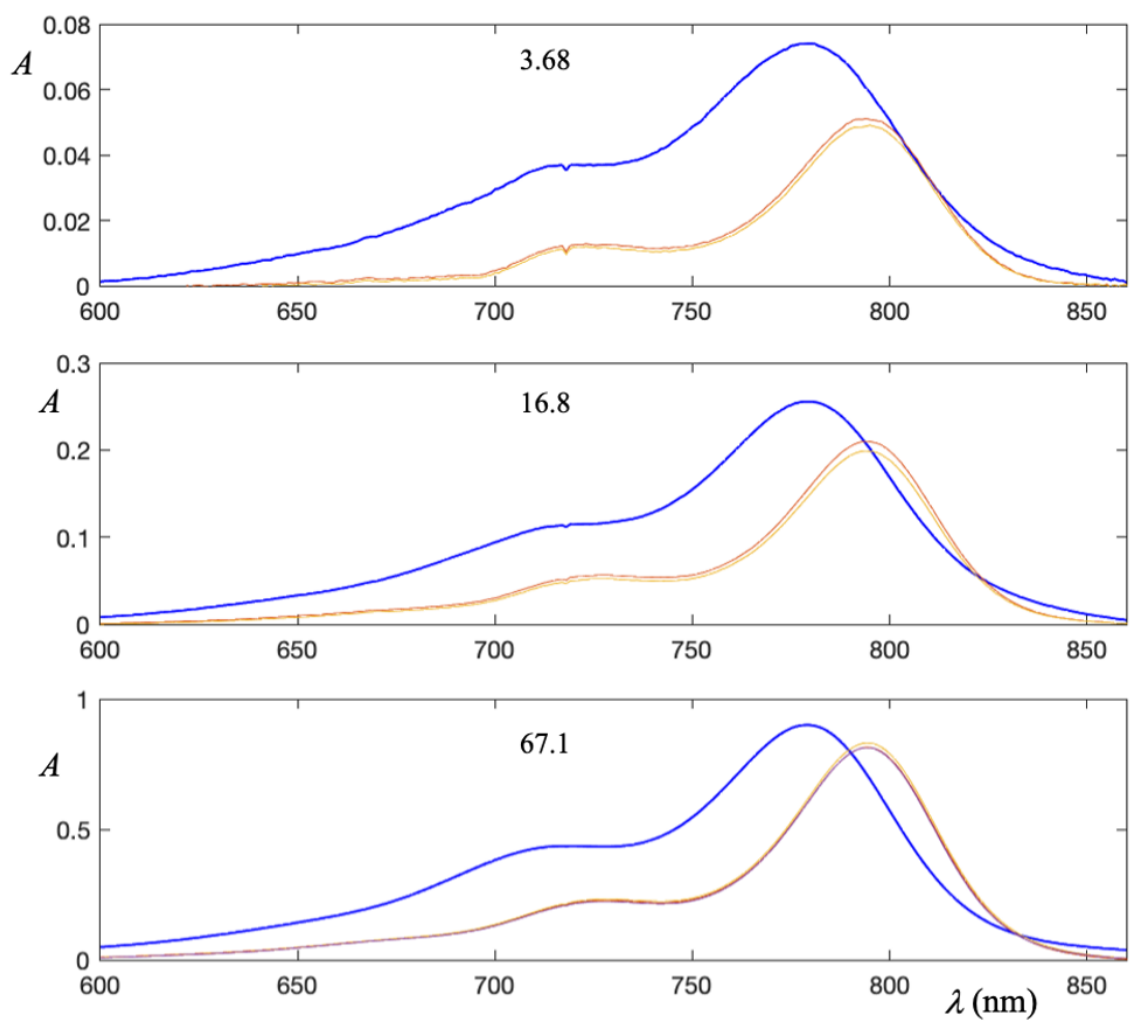

Figure 4. Absorption spectra of ICG in ternary systems LAP-ICG-water. In the presence of the clay absorption peaks are shifted towards right of about $13 \mathrm{~nm}$ (red shift). The number in the each plot is the ICG concentration expressed in $\mu \mathrm{M}$.

The aspects emerging from the spectra are essentially two. First, at each concentration of dye, the spectra acquired in the presence of 1, 2 or 3\% of LAP are superimposable. Second, the spectra in the ternary system undergo a marked red-shift of about 13-15 nm with respect to the corresponding spectrum in pure water (blue curves in Figure 4).

Generally, the absorption spectra are analyzed by monitoring the maximum absorption. Recently, some of us showed that this procedure can hardly capture information about the molecular structure and they defined the integral absorbance which allows us to generalize Beer's law. Mathematically it assume the following form

$$
\bar{A}(C)=\int_{\lambda_{1}}^{\lambda_{2}} A(\lambda, C) d \lambda
$$

where $\lambda$ is a wavelength (in $\mathrm{nm}$ ) within the experimental interval $\left[\lambda_{1}, \lambda_{2}\right]$ and $C$ is the molar concentration of the dye. Theì integral absorbance is proportional to the concentration of the absorbent species. It is intuitive that whenever solvation occurs in solution it has a marked effect on the thermodynamic properties of the solution. It is not quite so obvious that association effects, when occur, are also pf major importance. The reason for this is that the extent of association is a strong function of the composition, especially in the range which is dilute with respect to the associating component. On the other hand, Figure 4 highlights that absorption profiles overlap two spectra. This was confirmed by deconvolving the absorption profiles with two Gaussian peaks, as shown in Figure 5. The same procedure was applied to all measured absorption profiles. 


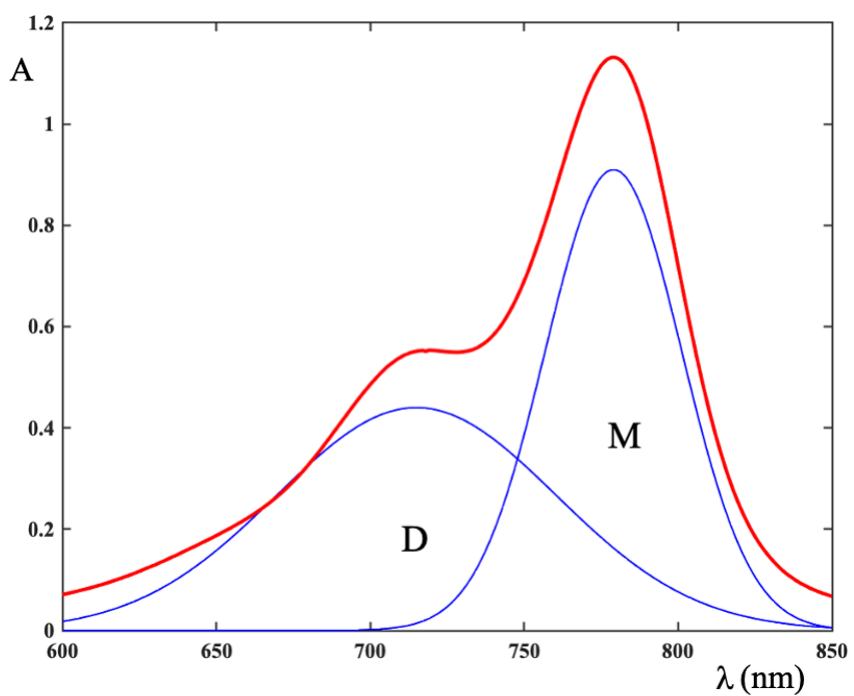

Figure 5. Monomer and dimer concentration calculated by the deconvolution of absorption spectra with two Gaussian peaks.

The total ICG concentration the dye-water system and in the ternary LAP-ICG-water system was the same, therefore by applying Equation (2) to all peaks, the monomer and dimer concentration in the individual systems is evaluated. The results are collected in Table 1.

Table 1. Concentration of the monomer and of the dimer calculated with the deconvolution of the spectra with two Gaussian peaks.

\begin{tabular}{cccc}
\hline $\begin{array}{c}C_{I C G} \\
(\mu \mathbf{M})\end{array}$ & $\begin{array}{c}W_{\mathbf{L A P}} \\
\mathbf{( \% )}\end{array}$ & $\begin{array}{c}C_{M} \\
(\mu \mathbf{M})\end{array}$ & $\begin{array}{c}C_{D} \\
(\boldsymbol{\mu M})\end{array}$ \\
\hline 67.1 & 0 & 33.9 & 16.6 \\
16.8 & 0 & 5.90 & 5.40 \\
3.68 & 0 & 1.28 & 1.20 \\
\hline 67.1 & 1 & 34.8 & 16.2 \\
16.8 & 1 & 9.40 & 3.70 \\
3.68 & 1 & 2.6 & 0.54 \\
\hline 67.1 & 2 & 34.8 & 16.2 \\
16.8 & 2 & 9.50 & 3.70 \\
3.68 & 2 & 2.70 & 0.49 \\
\hline 67.1 & 3 & 34.9 & 16.1 \\
\hline
\end{tabular}

Table 1 shows that, for a fixed ICG concentration, the monomer concentration in the ternary system is greater than in the corresponding binary system containing the same dye concentration. Correspondingly, the concentration of the dimeric species is reduced.

The ability of a molecule associate is closely related to its electronic structure. An ICG molecule has two nitrogen atoms, one positive, the other partially positive. As a result, the ICG molecule can anchor itself to the negatively charged LAP disk. Thus, a reasonable way to explain results of Table 1 is that association and binding equilibria take place in solution. Association refers to formation of dimers

$$
\mathrm{ICG}+\mathrm{ICG} \rightleftarrows \mathrm{ICG}_{2}
$$

Binding refers to formation of a chemical aggregate of two or more molecules which at least two are ICG and LAP

$$
\mathrm{LAP}+\mathrm{ICG} \rightleftarrows \mathrm{LAP}-\mathrm{ICG}
$$


In the binary system ICG-water only equilibrium (3) can occur, on the contrary in the ternary system LAP-ICG-water both equilibria take place. This implies that equilibrium (4) removes monomer from the dimerization process and, by virtue of Le Chatelier's principle, part of the dimer breaks down to form monomer. In other words, the total amount of dimer is reduced.

The empirical formula of the LAP-RD is known to be $\mathrm{Na}_{0.7}^{+}\left[\mathrm{Si}_{8} \mathrm{Mg}_{5.5} \mathrm{O}_{20}(\mathrm{OH})_{4}\right]^{-0.7}[1]$. Based on this formula, the molecular mass of the LAP is $762 \mathrm{~g} \cdot \mathrm{mol}^{-1}$ while the molecular mass of the ICG is $776 \mathrm{~g} \cdot \mathrm{mol}^{-1}$.

As it is seen, the molecular masses are very similar so that $1 \%$ of LAP represents a large excess in molecular terms. Moreover, equilibrium establishes that, in the presence of large LAP excess, the amount of complex produced depends only on ICG concentration. Hence, if ICG concentration is kept fixed in the experiment, the absorption spectra acquired at 1, 2 and $3 \%$ of LAP overlap.

The positive charge on the nitrogen atoms makes the LAP-ICG interaction stable enough to hold the molecules close together. Accordingly, their molecular orbitals split into bonding and anti-bonding pairs. Thus, the molecule is more stable and the resulting transition energy red shifted.

In Figure 6 absorption spectra of ICG are displayed, for a fixed LAP concentration. As it is immediately seen, maxima of absorption bands do not undergo shifts, reiterating that it depends on the LAP concentration. Furthermore, the area subtended by each spectrum depends not only on ICG concentration, but also on constants of equilibria (3) and (4). We proved that integral absorbance is given by following expression

$$
\bar{A}(C)=\overline{\epsilon_{1}} \ell[\mathrm{ICG}]+\overline{\epsilon_{2}} \ell[\mathrm{ICG}]^{2}+\overline{\epsilon_{3}} \ell[\mathrm{ICG}-\mathrm{LAP}]
$$

where [...] is the molar concentration, $\bar{\epsilon}_{i}$ is the integral molar extinction coefficient and $\ell$ is the light path length.

$$
\begin{gathered}
{\left[\mathrm{ICG}_{2}\right]=K_{1} \cdot[\mathrm{ICG}]^{2}} \\
{[L A P-I C G]=K_{2} \cdot[\mathrm{LAP}][\mathrm{ICG}]}
\end{gathered}
$$

Using the method described in detail in reference [21], the dye concentration is given by

$$
C=\frac{-\Gamma+\sqrt{\Gamma^{2}+8 K_{1} C}}{4 K_{1}}
$$

where $\Gamma=1+K_{2}[\mathrm{LAP}]$ has been used. obtaining

We assume $\frac{8 K_{1}}{\Gamma^{2}} C \ll 1$, so that Equation (8) can be developed into Taylor series,

$$
\begin{gathered}
{[\mathrm{ICG}]=\frac{\mathrm{C}}{\Gamma}+\ldots} \\
\bar{A}=\frac{\overline{\epsilon_{1}}+\overline{\epsilon_{3}} K_{3}[\mathrm{LAP}]}{\Gamma} \mathrm{C}+\frac{2 K_{1} \overline{\epsilon_{1}}}{\Gamma} C^{2}
\end{gathered}
$$

If the LAP concentration is kept constant in the experiment, the Equation (10) predicts a parabolic relationship between $\bar{A}$ the dye concentration. It is worth noting that the quadratic term in Equation (10) is positive, i.e.,

$$
2 \frac{K_{1} \bar{\epsilon}_{1}}{\Gamma}>0
$$

Equation (11) means that the function $\bar{A}(C)$ turns its concavity upward. Experimental results, plotted in Figure 7, seem confirm the model prediction. Moreover, the fitting parameters of each curve of Figure 7, i.e., for each LAP concentration, allow to estimate $K_{1}$. 

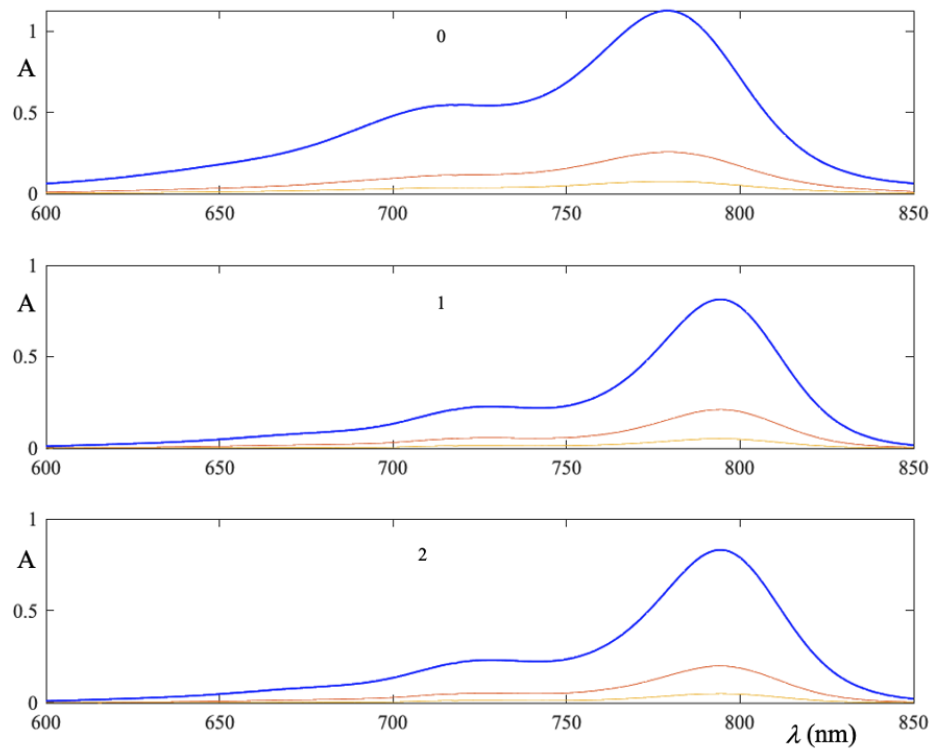

Figure 6. Absorptionspectrum in ternary LAP-ICG-water system with constant LAP mass fraction. By varying the ICG concentration, the absorption peaks do not shift, they only decrease in intensity. The number in the plot indicates the percentage $(\mathrm{m} / \mathrm{m})$ of LAP.

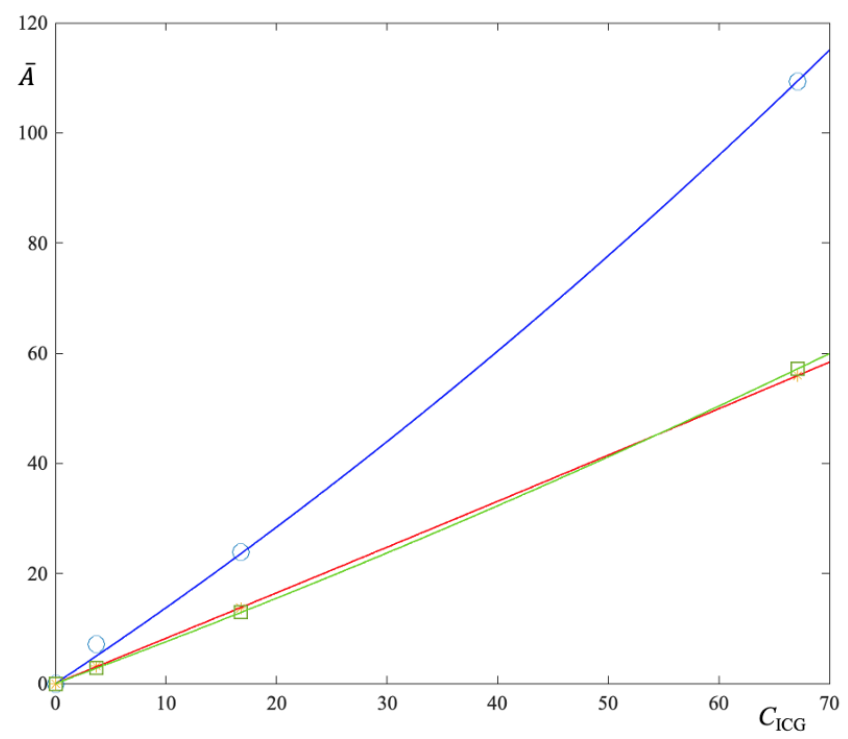

Figure 7. Integral absorbance as a function of ICG concentration for three fixed LAP concentrations $(1 \%, 2 \%$ and $3 \%)$. Numerical analysis reveals that experimental data are best-fitted by a polynomial of degree 2. This confirm the prediction of Equation (10).

\section{Conclusions}

The problem of delivering a vital dye toward a particular cells with a nano-carrier, is identical in certain respect to deliver cancer-drug. To address this problem, it is necessary to know which interactions are involved in the association/aggregation process to create the carrier nanocavities. The LAP ability to associate in water was monitored by $\mathrm{dz}$ measurements. The plot of $d_{z}$ vs. WLAP shows that LAP aggregates, however up to $3 \%(\mathrm{~m} / \mathrm{m})$ does not give rise to three-dimensional networks. The experimental results extrapolated to $W_{L A P}=0$ provide a value of $\mathrm{dz}$ very close to the diameter of the single LAP disk. Thus, in conditions of high dilution the LAP is completely exfoliated. The plot of $d_{z}$ vs. $C_{I C G}$, in the LAP-ICG-water ternary system with fixed $W_{L A P}=2 \%$, shows a saturation trend. Interestingly, $\mathrm{dz}$ extrpolated to $C_{I C G}=0$ is significantly smaller than in pure water at the same mass percentage. The absorption spectra of ICG in the ternary 
system LAP-ICG-water, acquired, at a fixed concentration of ICG, and for $W_{L A P}=1,2$, and $3 \%$ are perfectly superimposable. Furthermore the absorption peaks, with respect to the spectrum in water, shift in the red of about $10-13 \mathrm{~nm}$. Furthermore, if $W_{L A P}$ is fixed and the $C_{I C G}$ is varied, the absorption spectra do not undergo any spectral shift, but only decrease in intensity due to a decrease in $C_{I C G}$. It was shown that IGG in water exists predominantly as monomeric and dimeric specie. With this in view, the deconvolution of the absorption spectra by two Gaussian peaks, was applied for determining both the monomer and dimer concentration. Results show that, in the ternary system, increasing the ICG concentration reduces the dimer amount and increases that of the monomer. These experimental evidences are interpreted by means of synergy between the dimerization equilibrium and a LAP-ICG binding equilibrium. Obviously, in the ICG-water binary system only the dimerization equilibrium can exist, then the monomer and dimer concentration is uniquely determined by its equilibrium constant. In the ternary system, both equilibria exist and the concentrations of the dimer and the monomer are regulated by their simultaneous presence. By virtue of the Le Chatelier principle, if the monomer binds to the LAP discs, a certain dimer amount must be broken to restore a new dimerization equilibrium. Therefore the dimer concentration is reduced.

Author Contributions: Data curation, L.A.; investigation G.C.; methodology, G.C., F.L., G.B. and L.A.; project administration, L.A. All authors have read and agreed to the published version of the manuscript.

Funding: This research received no external funding.

Acknowledgments: The authors thank Giuseppe D'Amato for the IT support during the drafting of the paper.

Conflicts of Interest: The authors declare no conflict of interest.

Sample Availability: Samples of the compounds were provided from the authors.

\author{
Abbreviations \\ LAP Laponite \\ ICG Indocyanine green \\ DLS Dynamic Light Scattering
}

The following abbreviations are used in this manuscript:

\title{
References
}

1. Jatav, S.; Joshi, Y.M. Chemical stability of Laponite in aqueous media. Appl. Clay Sci. 2014, 97, 72-77. [CrossRef]

2. Tomás, H.; Alves, C.S.; Rodrigues, J. Laponite ${ }^{\circledR}$ : A key nanoplatform for biomedical applications? Nanomed. Nanotechnol. Biol. Med. 2018, 14, 2407-2420. [CrossRef] [PubMed]

3. Felbeck, T.; Behnke, T.; Hoffmann, K.; Grabolle, M.; Lezhnina, M.M.; Kynast, U.H.; Resch-Genger, U. Nile-Red-nanoclay hybrids: Red Emissive optical probes for use in aqueous dispersion. Langmuir 2013, 29, 11489-11497. [CrossRef] [PubMed]

4. Cinelli, G.; Cuomo, F.; Ambrosone, L.; Colella, M.; Ceglie, A.; Venditti, F.; Lopez, F. Photocatalytic degradation of a model textile dye using Carbon-doped titanium dioxide and visible light. J. Water Process Eng. 2017, 20, 71-77. [CrossRef]

5. Tabaraki, R.; Nateghi, A.; Yousefi, S.; Ahmady-Asbchin, S. Application of chemometric methods in modeling of competitive multidye biosorption from ternary system. J. Iran. Chem. Soc. 2017, 14, 285-296. [CrossRef]

6. Azad, F.N.; Ghaedi, M.; Asfaram, A.; Jamshidi, A.; Hassani, G.; Goudarzi, A.; Azqhandi, M.A.; Ghaedi, A. Optimization of the process parameters for the adsorption of ternary dyes by Ni doped $\mathrm{FeO}(\mathrm{OH})-\mathrm{NWs}-\mathrm{AC}$ using response surface methodology and an artificial neural network. RSC Adv. 2016, 24, 19768-19779. [CrossRef]

7. Cuomo, F.; Venditti, M.; Cinelli, G.; Ceglie, A.; Lopez, F. Olive mill wastewater (OMW) phenol compounds degradation by means of a visible light activated titanium dioxide-based photocatalyst. Z. Phys. Chem. 2016, 230, 1269-1280. [CrossRef]

8. Cheong, W.; Prahl, S.A.; Welch, A.J. A review of the optical properties of biological tissues. IEEE J. Quant. Electron. 1990, 26, 2166-2185. [CrossRef]

9. Philip, R.; Penzkofer, A.; Bäumler, W.; Szeimies, R.M.; Abels, C.A. Absorption and fluorescence spectroscopic investigation of indocyanine green. J. Photochem. Photobiol. A Chem. 1996, 96, 137-148. [CrossRef]

10. Ikagawa, H.; Yoneda, M.; Iwaki, M.; Isogai, Z. Tsujii, K. Yamazaki, R.; Kamiya, T.; Zako, M. Chemical toxicity of indocyanine green damages retinal pigment epithelium. Investig. Ophthalmol. Vis. Sci. 2005, 46, 2531-2539. [CrossRef] 
11. Di Nezza, F.; Zeppa, L.; Costagliola, C.; Bufalo, G.; Ambrosone, L. A physicochemical study of ophthalmological vital dyes: From dimerization equilibrium in buffer solution to their liposomal dispersions. Dye Pigments 2019, 162, 680-687. [CrossRef]

12. Jung, H.; Kim, H.-M.; Choy, Y.-B.; Hwang, S.-J.; Choy, J.-H. Itraconazole-Laponite: Kinetics and mechanism of drug release. Appl. Clay Sci. 2008, 1-4, 99-107. [CrossRef]

13. Jung, H.; Kim, H.-M.; Choy, Y.-B.; Hwang, S.-J.; Choy, J.-H. Laponite-based nanohybrid for enhanced solubility and controlled release of itraconazole. Int. J. Pharm. 2008, 349, 283-290. [CrossRef] [PubMed]

14. Viseras, C.; Cerezo, P.; Sanchez, R.; Salcedo, I.; Aguzzi, C. Current challenges in clay minerals for drug delivery. Appl. Clay Sci. 2010, 48, 291-295. [CrossRef]

15. Wang, S.; Wu, Y.; Guo, R.; Huang, Y.; Wen, S.; Shen, M.; Wang, J.; Shi, X.; Laponite nanodisks as an efficient platform for doxorubicin delivery to cancer cells. Langmuir 2013, 29, 5030-5036. [CrossRef]

16. Di Biasio, A.; Ambrosone, L.; Cametti, C. Numerical simulation of dielectric spectra of aqueous suspensions of non-spheroidal differently shaped biological cells. J. Phys. D Appl. Phys. 2008, 42, 025401. [CrossRef]

17. Ambrosone, L.; Sartorio, R.; Vescio, A.; Vitagliano, V. Volumetric properties of aqueous solutions of ethylene glycol oligomers at $25^{\circ}$ C. J. Chem. Soc. Faraday Trans. 1996, 92, 1163-1166. [CrossRef]

18. Jung, B.; Vullev, V. I.; Anvari, B. Revisiting indocyanine green: Effects Of serum and physiological temperature on absorption and fluorescence characteristics. IEEE J. Sel. Top. Quant. Electron. 2016, 20, 149-157. [CrossRef]

19. Xiang, J.; Chen, Z.; Yan, Y.; Ai, X.; Liu, Y.; Xu, G. Photoinduced electron transfer from the excited J-aggregate state of a thiacarbocyanine dye to $\mathrm{TiO}_{2}$ colloids. J. Colloids Interface Sci. 2002, 254, 195-199. [CrossRef]

20. Bujdák, J.; Iyi, N.; Fujita, T. Aggregation and stability of 1,1'-diethyl-4, $4^{\prime}$-cyanine dye on the surface of layered silicates with different charge densities Colloids Surf. A Physicochem. Eng. Asp. 2002, 1-3, 207-214.

21. Di Nezza, F.; Guerra, G.; Costagliola, C.; Zeppa, L.; Ambrosone, L. Thermodynamic properties and photodegradation kinetics of indocyanine green in aqueous solution Dye Pigments 2016, 134, 342-347. [CrossRef] 